\section{“iNo es esto, no es esto!”: reflexiones docentes desde una universidad presencial (presuntamente) virtualizada1}

\section{Carlos Menéndez Otero \\ Universidad de Oviedo}

En diciembre de 2018, la Revista de Lenguas para Fines Específicos publicó nuestro artículo "Ensuring uniformity of content and assessment in multi-section, on-site ESP university courses through ICT". El texto realiza un análisis crítico del Proyecto de Innovación Docente (PINN) que desarrollamos durante el curso 2016-17 en Inglés para el Ámbito Comercial I, una asignatura presencial cuatrimestral de lengua inglesa para fines específicos (IFE) del Grado en Comercio y Marketing de la Universidad de Oviedo (en adelante, UniOvi), y que tuvo entre sus objetivos principales la informatización de la evaluación continua de la asignatura. Lejos del triunfalismo hegemónico en la literatura, el artículo reconoce los beneficios de la integración de las TIC en la enseñanza, pero advierte de que el tiempo de preparación de una prueba de evaluación electrónica es mucho mayor que el de una en papel, que su dependencia de factores no controlables por el docente convierten su celebración en algo imprevisible y, sobre todo, que la actitud-y aptitud —ejemplar de estudiantes e instituciones académicas hacia estas pruebas y las TIC en general tiene más de mito que de realidad. De hecho, la conclusión incide en la última advertencia a través de la siguiente reflexión:

$[\ldots]$ the experience shows that some students welcome the use of ICT in the classroom simply because they erroneously assume that computer-based tests are much easier than paper-based ones, and feel bitterly disappointed when they find out that this form of assessment can be as rigorous [...] Closely related to this is the fact that most Generation Z students just use ICT for social networking (i.e., recreational purposes) and, as the experience bears out, lack or have a very limited knowledge of many digital skills considered essential in today's workplace.

Teachers should not take for granted the digital skillset of their digital-native students. However, for these skills to be effectively integrated into curricula and syllabi much greater effort at institutional level is also necessary. Thirdlevel educational innovation cannot thrive without appropriate institutional support in the form of greater flexibility in procedures and structures, acknowledgment and incentives for innovative teachers [...], and especially substantial, continuous investment. Most universities in Spain, including ours, boast their commitment to educational innovation. However, having had to put up with scarce, cramped computer labs; malfunctioning PCs, and often unreachable, unresponsive VLE administrators, we can only wonder whether this commitment is real or simply rhetorical. Maybe it is real-as long as faculty members are willing to carry the burden of innovation on their own. (Menéndez-Otero, Serrano-González y Gil-Naveira, 2018: 23)

Si traemos este artículo a colación es porque, a nuestro juicio, la accidentada respuesta de UniOvi al reto de continuar la actividad docente durante el confinamiento por la pandemia de la COVID-19 no ha tenido tanto que ver con la enormidad sin precedentes del reto como con que, a 13 de marzo de 2020, fecha de suspensión de las clases presenciales, los problemas de digitalización expuestos, así como otros muchos, se amontonaban bajo las alfombras en espera de solución.

${ }^{1}$ El entrecomillado hace referencia a una de las citas más famosas de Ortega y Gasset (1931).

\title{
MAGISTER
}

Vol. 32. Núm. I: (2020). Sección extraordinaria 
Lejos de tratar de solucionar viejos o nuevos problemas, o admitir ante la sociedad que, sin financiación adicional, muchas cosas se tendrían que dejar de hacer durante el confinamiento por falta de medios, a partir del 13 de marzo, parafraseando una frase de Top Gun (Tony Scott, 1986), UniOvi extendió cheques de docencia virtual que no podía pagar y pasó los cargos al personal docente e investigador (PDI) en dos (in)cómodos plazos. Con el primero, el ya elevado número de horas invisibles de dedicación docente del PDI aumentó de forma exponencial. El segundo fue aún más allá y, en la práctica, expropió gratuita y temporalmente los dispositivos electrónicos, conexión a Internet, suministro eléctrico, material fungible, mobiliario y habitaciones particulares del PDI para ponerlos al servicio de la institución. ${ }^{2}$ Los muchos problemas de hardware, software, conectividad y ergonomía derivados del teletrabajo no fueron, sin embargo, expropiados y tanto su rápida subsanación como los costes derivados de ella siguieron siendo en todo momento responsabilidad particular del PDI.

En cierto modo, con las labores de investigación del PDI y la carrera profesional que de ellas depende ocurrió algo parecido, aunque escasamente sorprendente si tenemos en cuenta lo que ha venido sucediendo desde que alguien decidió que se podía implantar con éxito el Plan Bolonia, un sistema de educación superior pensado por y para centros de élite, en universidades masificadas e infrafinanciadas como UniOvi. El coste cero al que se ha pretendido implantar este plan ha hecho que tareas como la coordinación y evaluación de asignaturas, la participación en tribunales de Trabajos Fin de Grado (TFG) y Máster, y la dirección de PINN, entre otras, mantengan una asignación de cero horas en unos planes de ordenación docente que, por otra parte, también asumen interesadamente que un TFG se puede dirigir en cuatro horas y que un grupo de 35 estudiantes de grado exige la misma dedicación que uno de cinco. Que todo esto pueda caber en las 240 horas de dedicación docente anual de buena parte del PDI no extraña, sin embargo, a las comisiones de

2 Ante la posibilidad de que el curso 2020-21 sea también online, sería aconsejable que las universidades presenciales hiciesen la reflexión que planteaba Josep A. Planell, rector de la UOC, en junio de 2020 en el diario $E$ País:

No puedes enviar a casa a los trabajadores y decir "ya te apañarás". Hay que darles los instrumentos. La gente ahora sillas porque no tienen una para hacer una jornada de ocho horas. Hemos vivido una emergencia y se acreditación de ANECA ni a las de plazas de cualquier universidad, incluida la propia UniOvi. Lo que extraña-y, por tanto, penaliza cada vez más en un contexto de creciente endurecimiento de los baremos de acreditación y acceso a plazas - es que, con supuestamente tanto tiempo libre para investigar, nuestra producción científica no sea tan extensa como la del PDI de universidades con un reconocimiento docente más realista.

Así pues, el problema no es tanto que la docencia virtual haya supuesto un importante esfuerzo adicional de dedicación docente que no va a tener reconocimiento curricular como que tal esfuerzo se haya exigido en un contexto donde ya eran muchos los esfuerzos adicionales sin reconocimiento. Cuando nos presentemos a la próxima acreditación o plaza, a la comisión le dará igual si durante estos meses no hemos podido elaborar ningún artículo, capítulo o comunicación porque hemos tenido que dedicar una media de doce horas diarias, los siete días de la semana, fines de semana, festivos y vacaciones incluidos, a atender cuestiones derivadas del forzoso intento de transformación, de un día para otro y a coste cero, de la docencia presencial de UniOvi en virtual. También le dará igual si, una vez terminada la docencia virtual, a consecuencia del sobreesfuerzo, nuestra salud física o psicológica se ha resentido y hemos de aguardar todavía un tiempo antes de poder retomar nuestra producción científica con normalidad. Y una y otra cosa le darán igual porque UniOvi nunca reconocerá este enésimo esfuerzo adicional de forma oficial, y ni ella, ni ANECA, ni ninguna otra universidad española aprobará jamás un baremo compensatorio que lo tenga en cuenta. ${ }^{3}$

Si hemos dicho "intento" de virtualización es porque, a pesar del tiempo y esfuerzo invertidos, como afirma Josep A. Planell, lo hecho estos meses en las universidades presenciales puede haber evitado el colapso, pero no puede llamársele educación online. El propio rector de la UOC apunta que, mientras las universidades

ha tenido que solventar como se ha podido, pero si esto lo queremos hacer bien, hay que replantear muchas cosas. (Vallespín, 2020)

${ }^{3}$ De hecho, en vista del borrador de Estatuto del PDI que trascendió a comienzos del verano de 2020, parece casi que, bajo la excusa de mejorar las condiciones laborales de los Profesores Asociados, el Ministerio de Universidades pretende "recompensar" este esfuerzo con nuevas trabas al acceso a los cuerpos docentes universitarios y una mayor precarización del conjunto del PDI laboral.

\section{MAGISTER}

Vol. 32. Núm. I: (2020). Sección extraordinaria 
presenciales se han empeñado en poner al profesorado frente a una webcam "[para transmitir] su conocimiento", en un modelo verdaderamente online como el de la UOC, "no hay clases presenciales, el estudiante aprende haciendo" (Vallespín, 2020). Dicho de otro modo, al igual que ocurrió cuando, a finales de los años 90, la prensa creyó ingenuamente que se podía hacer la edición digital de un periódico con el mero volcado de contenidos de la de papel, en la pandemia de 2020, las universidades presenciales asumieron erróneamente que hacer enseñanza online consistía en poner al PDI delante de una webcam en el horario previsto para la docencia presencial y que continuara impartiendo sus clases como si nada hubiese ocurrido.

A título personal, tuvimos claro desde un primer momento que ni esto era educación online ni UniOvi tenía capacidad técnica o humana para poder llevarlo a cabo con éxito. Entre otras razones, quizá la más importante era que, al prescindir de uno de los principios esenciales de la educación online (i.e., la supresión de las barreras de tiempo y espacio para el aprendizaje), las conexiones al Campus Virtual (CV) de miles de estudiantes y profesores se iban a concentrar en unas horas muy determinadas y, más aún, iban a hacerlo generando un pesado tráfico de audio y vídeo. Que el CV fuese capaz de gestionar correctamente este volumen de datos nos parecía tan poco probable como que el alumnado centennial, que abandona los vídeos de YouTube a los 2-3 minutos, estuviese dispuesto a soportar una sucesión de talking heads (o, en su defecto, emisiones de audio) durante horas, que todo el profesorado supiese utilizar aplicaciones de videoconferencia, y que tanto profesores como alumnos contasen en sus domicilios con equipos y conexiones capaces de emitir y recibir audio y vídeo de forma fluida. Por último, por experiencia sabíamos que, a pesar de lo dicho en los mensajes de las autoridades universitarias, tampoco iba a haber un equipo de soporte capaz de solucionar de forma rápida los problemas y dudas técnicas que surgieran.
Tal y como esperábamos, a ciertas horas, el exceso de tráfico no tardó en hacer prácticamente imposible cualquier intento de (video)conferencia a través del CV y originó problemas en la subida y actualización de contenidos y calificaciones, el inicio y cierre de sesión, y el reenvío automático de mensajes al correo del alumnado, así como caídas frecuentes del sistema. Por su parte, la activación de Microsoft Teams en la intranet de UniOvi, aunque rebajó algo el tráfico del CV conforme fue creciendo su uso, no fue tampoco la panacea que se anunciaba porque la comunidad universitaria no tuvo realmente tiempo para aprender a manejar la aplicación con soltura, la calidad de las videoconferencias no fue la esperada, y la adición de un nuevo canal de tareas y mensajería generó un notable grado de confusión entre profesores y alumnos.

En el momento de suspensión de la actividad presencial, nos encontrábamos impartiendo los tres grupos de prácticas de aula de la asignatura cuatrimestral Inglés II: Documentación Profesional para el Turismo, correspondiente al Grado en Turismo y con un total de 73 alumnos matriculados. ${ }^{4}$ Esta asignatura de IFE se centra en la competencia escrita, $\mathrm{y}$ tiene como objetivo prioritario que el alumnado adquiera las funciones, gramática y, sobre todo, vocabulario específico necesarios para elaborar con un mínimo de corrección algunos de los documentos más comunes en el sector turístico. Distribuida en cuatro temas, la materia se trabaja en las sesiones prácticas presenciales a partir de una serie de materiales impresos que, justo antes del inicio de cada tema, depositamos en el servicio de reprografía. Estos materiales contienen actividades orientadas a la introducción, práctica y consolidación de los contenidos del curso, que en parte pueden ser también trabajados de forma autónoma a través de los recursos electrónicos que proporcionamos en el CV. Las soluciones de cada actividad se suelen discutir oralmente en clase, aunque también recogemos periódicamente actividades para corregir y, con ello, comprobar la evolución individual del alumnado. ${ }^{4}$ Por razones de espacio, queda fuera de esta reflexión el importante volumen de trabajo que, en paralelo, generó la
docencia y coordinación de asignaturas de primer cuatrimestre en forma de consultas por correo electrónico,
modificación de Guías Docentes, y preparación, corrección y revisión de los exámenes online de mayo y junio.

\section{MAGISTER}

Vol. 32. Núm. I: (2020). Sección extraordinaria 
Con el fin de incitar a los estudiantes a estudiar los contenidos y asistir a las clases regularmente, la convocatoria ordinaria (mayo) se vincula a la evaluación continua (60\%) y consta de dos pruebas escritas (25\% cada una), dos tareas sorpresa (5\% cada una) y un examen final (40\%) con dos partes, una con ejercicios de vocabulario y gramática (20\%), y otra en la que deben elaborar alguno de los documentos vistos a lo largo del curso (20\%). El doble objetivo anterior se completa con la exigencia de notas medias mínimas de 4 puntos entre las dos pruebas de evaluación continua para poder hacer el examen final, y entre las dos partes del examen para que éste haga media con el resto de las pruebas y tareas. Por su parte, la convocatoria extraordinaria consta de un único examen final (100\%), en el que los alumnos deben resolver cuestiones de gramática y vocabulario específicos (40\%), elaborar alguno de los documentos vistos durante el curso (40\%), y responder a preguntas de comprensión sobre algún documento turístico $(20 \%)$. En un intento por reproducir las condiciones de la evaluación continua, el alumnado debe alcanzar una calificación mínima de 4 puntos en cada una de las dos primeras partes del examen para que la tercera sea tenida en cuenta.

En línea con cursos anteriores, en el momento de suspensión de la actividad presencial, se registraba una importante discrepancia entre el porcentaje de presentados a la primera prueba de evaluación continua (64, un $87,7 \%$ del total) y el de asistencia regular a clase (40-50\%), así como un notable grado de correlación entre la falta de asistencia y la obtención en la prueba de una calificación inferior a 5 puntos (42). Aun cuando para hacer el examen final de mayo se exige una media de 4 puntos entre las dos pruebas de evaluación continua, lo habitual es que casi todos los que obtienen una calificación igual o inferior a 3 en esta prueba no se presenten ya a la siguiente (en este caso, 15), que una mayoría de los que tienen calificaciones superiores a 4 se presenten a la segunda y lleguen al examen de mayo (40), y que unos pocos de los que tienen calificaciones comprendidas entre 3 y 4 (11) se presenten también a la segunda prueba y, además, consigan la puntuación suficiente para hacer el examen. Esto es así porque, sin un adecuado conocimiento del vocabulario específico en que se centra la primera prueba, resulta prácticamente imposible elaborar ninguno de los documentos que pedimos en la segunda. De haber continuado las clases con normalidad, por tanto, lo esperable habría sido un máximo de 45-50 alumnos en la segunda prueba y de 35-40 en el examen final de mayo.
Teniendo en cuenta que la asistencia siempre desciende en la segunda mitad del cuatrimestre y que las tareas sorpresa solo están abiertas a quien asiste regularmente a clase, en condiciones normales, un máximo de 36 alumnos habría llegado a hacer la primera y uno de 29, la segunda. Podemos añadir a estas previsiones que lo habitual es que el $90-95 \%$ de los presentados en mayo superen la asignatura en la convocatoria ordinaria y el resto lo haga en la extraordinaria.

En un intento por sortear los problemas antes expuestos y ofrecer a los alumnos algo que pudiera parecerse a una verdadera enseñanza online, contribuyera a desarrollar sus competencias digitales y de inglés escrito, evocara la dinámica cooperativa de las prácticas presenciales $\mathrm{y}$, sobre todo, permitiera alcanzar los objetivos de aprendizaje previstos, asentamos la docencia no presencial en tres tipos de actividades asincrónicas de Moodle: foros de discusión, tareas y glosarios. Aunque en algunas pudimos utilizar ejercicios de los materiales impresos, para una mayoría tuvimos que, bien hacer una adaptación de los anteriores, o bien crear exprofeso nuevas actividades y recursos.

Si bien nos parecía improbable que los alumnos que no habían asistido hasta entonces con asiduidad a las clases presenciales fuesen a conectarse regularmente al $\mathrm{CV}$, desde el principio planteamos cada semana nuevas actividades, off- y online, en un foro en inglés llamado "Activities instructions" y les asignamos plazos de realización comprendidos, en general, entre los dos y los cuatro días. A lo largo de todo el período no presencial, cada nueva actividad implicó la apertura de un nuevo hilo en el foro. Mediante el título y primera entrada de cada hilo, los estudiantes podían saber rápida y claramente el plazo e instrucciones de realización, los recursos que debían utilizar, si la actividad era o no evaluable, si era individual o grupal, y si era para realizar de forma autónoma o preveía el envío de output al hilo o a algún otro espacio del CV.

Huelga decir que, independientemente del tipo de actividad, los alumnos podían plantear sus dudas sobre cada una de ellas en el propio hilo, por correo electrónico o mediante la aplicación de mensajería de Moodle. No obstante, tomamos varias medidas adicionales para evitar malentendidos y excusas oportunistas para la no

\section{MAGISTER}

Vol. 32. Núm. I: (2020). Sección extraordinaria 
realización o realización incorrecta de las actividades. En primer lugar, mantuvimos en paralelo un segundo foro en castellano ("Course noticeboard"), en el que, entre otras informaciones, preanunciamos una mayoría de actividades dando indicación de día y hora aproximados de su apertura y plazo de realización, recordamos la inminencia del cierre de algunos plazos y ofrecimos explicaciones suplementarias de muchas instrucciones. En segundo, a la hora de diseñar el calendario, tuvimos presente que las primeras actividades del período no presencial debían contribuir a crear en los estudiantes el hábito de conectarse regularmente al CV y que, en general, cada una de las actividades evaluables que propusiésemos debía ir precedida de varias similares de carácter no evaluable, en las que pudiesen practicar sin temor a repercusiones en su calificación. En tercero, aunque todas las actividades podían realizarse y entregarse desde un dispositivo y conexión a Internet básicas, nos aseguramos de que las individuales exigieran únicamente la introducción de una palabra y/o unas pocas frases en el propio foro o en un glosario y que, por tanto, pudieran ser realizadas sin dificultad desde un teléfono móvil. Por último, en un intento por evitar la repetición de errores y favorecer el aprendizaje, proporcionamos un feedback individualizado a todas las entradas de foro y ficheros entregados por los alumnos, evaluables y no evaluables, creamos y subimos al CV solucionarios y explicaciones teóricas en PDF de las actividades de realización autónoma, y ofrecimos feedback general en castellano del conjunto de actividades en el foro "Course noticeboard".

A pesar de todas estas precauciones y de que, ya al principio del período no presencial, advertimos de que las actividades online nunca supondrían más de un $40 \%$ de la calificación final, los problemas técnicos, la expectativa de que una asignatura online es por definición mucho más fácil, las carencias lectoras de parte del alumnado y, sobre todo, la inconcreción normativa de UniOvi en lo relativo a la evaluación online dificultaron la relación con los estudiantes y nos obligaron a hacer aún mayores esfuerzos de corrección y explicación.

\footnotetext{
${ }^{5}$ En nuestra opinión, la propuesta más descabellada de las planteadas en UniOvi en torno al 13 de marzo fue la de que se impartieran las clases online desde los despachos. Por fortuna, la declaración del estado de alarma hizo totalmente inviable tal posibilidad.
}

\section{MAGISTER}

Vol. 32. Núm. I: (2020). Sección extraordinaria
Las dos primeras semanas de confinamiento transcurrieron en medio de rumores de vuelta a la actividad presencial en abril e, incluso, propuestas descabelladas para que el PDI, por ejemplo, no solo tuviera que impartir online las clases previstas durante esos días, sino que tuviera que hacerlo también otra vez al final del cuatrimestre de forma presencial. ${ }^{5}$ Con el propósito de fomentar la participación en las actividades online sin comprometernos realmente a nada, comenzamos el período proponiendo discusiones online "potencialmente evaluables" dentro del 10\% de tareas sorpresa, en las que los estudiantes debían responder una serie de cuestiones en el propio hilo dentro de un plazo. De cara a asegurar la participación del máximo posible de alumnos, potenciar el aprendizaje colaborativo y reducir la cantidad de mensajes, se pidió a los estudiantes que intervinieran una sola vez por hilo de discusión y que solo respondieran a preguntas ya respondidas si estimaban que las respuestas existentes en el hilo eran incorrectas. Puesto que algunos estudiantes se quejaron de que había un pequeño grupo que monopolizaba las conversaciones, pronto garantizamos que, de contar estas actividades en la calificación final, solo lo haría un máximo de una intervención correcta en el conjunto de las discusiones y comenzamos a pedir de forma reiterada que quien tuviera ya una intervención correcta se abstuviera de intervenir. Asimismo, para asegurarnos de que los 73 matriculados pudiesen tener al menos una intervención correcta, procuramos que el número total de preguntas fuera muy superior al de estudiantes y, además, diseñamos muchas de ellas para que tuvieran más de una respuesta posible.

Aunque continuamos utilizando los foros de discusión durante todo el período no presencial, el último hilo "potencialmente evaluable" se cerró el 27 de marzo. A pesar del elevado número de ítems para responder y de que hubo varios hilos donde todas las respuestas fueron incorrectas, muchos estudiantes continuaron alegando durante semanas que no habían tenido oportunidad de intervenir por culpa de otros, que no lo habían podido hacer a tiempo porque el CV no les había reenviado los mensajes de apertura de los distintos hilos, o que la misma conexión desde la que se estaban quejando no les había permitido, paradójicamente, intervenir a tiempo en ningún hilo. Otros se dedicaron durante toda la actividad a corta-pegar respuestas de 
sus compañeros o de nuestras correcciones, o a responder cosas que no tenían nada que ver con las instrucciones, sin sentido alguno, y/o que revelaban que no habían siquiera ojeado los materiales o recursos indicados para su resolución. Más aún, aprovechando la imposibilidad de bloquear en Moodle la adición de entradas a una discusión ya terminada, otros trataron de añadir nuevos posts horas, días e, incluso, semanas después del fin de la actividad. Podemos añadir asimismo que los registros de actividad en el CV de una mayoría de estos alumnos nos indicaron que casi todos pasaban muchos días sin conectarse y que, cuando lo hacían, iban directamente a alguno de los foros y, sin tiempo material para mirar ningún recurso ni leer el propio hilo, escribían y/o corta-pegaban una respuesta, y se desconectaban.

Muchos de estos comportamientos continuaron en la tarea explícitamente evaluable que propusimos para la semana del 20 al 27 de marzo - la adición de una entrada no repetida a un glosario bilingüe de términos de correspondencia comercial—y aun en las tres grupales ${ }^{6}$ que planteamos desde el 31 , una vez anunciado oficialmente que no habría más docencia presencial en lo que restaba de cuatrimestre y que, por tanto, al menos la evaluación continua se completaría online. En la continuidad de estos comportamientos tuvo mucho que ver el hecho de que, mientras el 31 de marzo pudimos establecer unas normas claras para la evaluación continua, casi todo lo relativo a los exámenes de mayo estuvo rodeado de un halo de provisionalidad e incertidumbre hasta el propio mayo. ${ }^{7}$ Como consecuencia, muchos estudiantes creyeron erróneamente durante todo el mes de abril que UniOvi terminaría por imponer un modelo de evaluación mucho más ventajoso para ellos-o, incluso, un aprobado general-y, por tanto, no hacía falta hacer (casi) nada para superar la asignatura.

Sea como fuere, el 31 de marzo confirmamos el calendario y los pesos de las distintas actividades online evaluables, que éstas tendrían en conjunto un valor máximo del

${ }^{6}$ En estas tres actividades, se pidió a los alumnos que, en grupos de hasta 3-4 personas, elaboraran una carta de respuesta a una petición de información para celebrar una boda en un hotel, una presentación sobre el diseño y creación de encuestas de satisfacción, y un cuestionario de evaluación de una atracción turística de Asturias, respectivamente.

${ }^{7}$ A mediados de abril, UniOvi recondujo hacia el PDI la creciente presión en redes sociales para que la institución aclarase qué ocurriría con los exámenes finales, y le exigió que comunicase oficialmente a los estudiantes qué
$40 \%$ en la calificación, que el de la prueba presencial realizada antes del confinamiento sería del $60 \%$, y que quien hubiese obtenido en ella una calificación igual o inferior a 3 podía contar como no presentado a la convocatoria ordinaria si nos lo comunicaba antes del 1 de mayo. Asimismo, especificamos que, ya fuera presencial u online, el examen de junio mantendría su estructura y condiciones de nota mínima originales. De forma adicional, concretamos que la intervención en las discusiones tendría finalmente un valor de 0,4 puntos y la entrada del glosario de 0,6 , es decir, como habíamos anunciado, tendrían en conjunto un peso equivalente al de las tareas sorpresa. Tal y como reiteramos el propio 31 de marzo y a lo largo de todo el mes de abril, lo único que no podíamos confirmar era si UniOvi permitiría que, como deseábamos, la calificación de mayo fuese la suma 60-40 antes mencionada o, por el contrario, tendríamos que mantener el examen final recogido en la Guía Docente, en cuyo caso el resultado de esa suma determinaría la calificación de la evaluación continua, los estudiantes con un mínimo de 4 tendrían que realizar el examen final y el resto tendría la condición de suspenso o no presentado en la convocatoria.

A diferencia de otros cursos, el primer escenario - que finalmente aprobó UniOvimantenía intactas las posibilidades de superar la asignatura en mayo para cualquier alumno con una calificación en la prueba presencial igual o superior a 1,25, aunque obviamente la posibilidad era tanto más remota cuanto más baja fuera esta calificación. Esta posibilidad, los rumores sobre posibles aprobados generales con condiciones muy flexibles, la indefinición oficial de UniOvi y la ciega fe de algunos en que el mero envío de las actividades les garantizaría el aprobado incrementaron la participación media en las actividades evaluables hasta los 54 alumnos (72\%). El esfuerzo de corrección y retroalimentación extra que supuso esta alta participación no se vio, sin embargo, reflejado en el de aprobados en la convocatoria ordinaria, que aun sin examen final se quedó en 32 alumnos $(42,1 \%)$, en su mayoría, personas

modelo de evaluación aplicaría en mayo y junio. Sin embargo, puesto que muchas de las comisiones encargadas de aprobar estos modelos no se reunieron hasta mayo, algunos de ellos terminaron por ser rechazados o modificados a muy pocos días del inicio del período de exámenes, con el consecuente daño a la credibilidad del profesorado.

\section{MAGISTER}

Vol. 32. Núm. I: (2020). Sección extraordinaria 
que habían asistido a las clases presenciales, se habían conectado con regularidad al CV, tenían una calificación de 4 o más en la prueba presencial, y un nivel de inglés general aceptable o bueno. A pesar de esta relativa continuidad con los datos de los cursos presenciales, cabe reseñar que, sorprendentemente, más de la mitad de los 19 alumnos con calificaciones en la prueba presencial entre 4 y 4,99, y una con una calificación superior a 5, terminaron suspendiendo la convocatoria por la deficiente realización, no realización y, en algún caso, plagio de una o más actividades online. En el extremo contrario, dos alumnas con notas inferiores a 4 en la prueba presencial superaron la asignatura en mayo.

A la publicación de las calificaciones de mayo siguió un aluvión de peticiones de explicaciones, reclamaciones e, incluso, exigencias reiteradas de repetición de actividades concluidas y revisadas hacía semanas por parte de alumnos suspensos que, en muchos casos, nos enviaron en apenas unos días un mayor número de correos que veces se habían conectado al CV en todo el cuatrimestre. ${ }^{8}$ A continuación, conforme se acercaba el examen final de junio, y muy particularmente en los días previos al mismo, se sucedieron las peticiones de envío por correo electrónico de los materiales impresos y electrónicos de la asignatura de alumnos que, a pesar de carecer de ellos, habían tratado de superarla en evaluación continua.

Como viene siendo habitual en los últimos años, el examen final de junio volvió a confirmar que, salvo que el nivel de inglés general sea demasiado bajo y/o el alumno haga un importante esfuerzo de estudio autónomo, una adecuada realización de la primera prueba de evaluación continua y la asistencia regular a clase son determinantes en la calificación final del curso. Desafortunadamente, el examen sirvió asimismo para confirmar una vez más las muchas carencias del CV y ofrecer al menos una estimación del porcentaje de fraude que podría estar registrándose en las pruebas online.

${ }^{8} \mathrm{~A}$ pesar de reclamar sin cesar la repetición de pruebas y tareas, algunos llegaron reconocieron en estos correos que habían acordado con sus compañeros figurar en varias actividades grupales donde, en realidad, no habían hecho nada.

\section{MAGISTER}

Vol. 32. Núm. I: (2020). Sección extraordinaria
En lo que respecta a las carencias, cabe reseñar que, a pesar del importante esfuerzo que supone la creación y configuración de un examen en Moodle, las únicas medidas de seguridad mínimamente efectivas parecen ser las de la limitación del tiempo de examen y el número de intentos por pregunta. El resto no serían más que una pérdida de tiempo que los alumnos pueden sortear con mucha facilidad. Así, pudimos constatar en este examen que, por ejemplo, el CV no impide que un mismo usuario tenga dos sesiones abiertas simultáneamente desde distintos dispositivos, que la opción de navegador seguro no bloquea la apertura de varias ventanas a la vez en una mayoría de navegadores y que, salvo que se tenga tiempo para crear un banco de preguntas realmente extenso, la aleatoriedad en el orden de preguntas es inútil si los alumnos acuerdan coordinarse para compartir respuestas por WhatsApp. La aplicación de videoconferencia BigBlueButton, desde la que se supone se debería poner hacer al menos una vigilancia disuasoria a través de webcam, no hace sino empeorar todas estas vulnerabilidades, pues permite acceder a cualquiera a una sala de videoconferencia, sea o no parte de la lista de usuarios predefinida, y es incapaz de gestionar simultáneamente más de 20-25 feeds de vídeo silente. Más aún, como nos ocurrió en este y otros exámenes, en el caso de que ocurran estos problemas de gestión de señal, suele ser habitual que la aplicación expulse al moderador de la sala y no le vuelva a dar acceso - si es que se lo llega a dar-mientras no disminuya el número de asistentes conectados.

Conscientes de todas estas cuestiones, reforzamos la seguridad de la prueba mediante la inclusión de preguntas donde solo el uso de Google Translate, el propio buscador Google o Wikipedia podía llevar a responder de una forma muy específica (y, además, errónea), así como ejercicios abiertos de análisis y desarrollo donde la semejanza entre respuestas de personas diferentes solo podía significar que estas personas se habían copiado entre ellas. Como resultado de estas medidas y el análisis de los registros del examen, pudimos determinar con absoluta seguridad que al menos 11 alumnos - es decir, el 32,3\% de los 34 presentados-habrían intentado superar la asignatura en junio haciendo uso de uno o varios de los métodos anteriores. Con todo, las discrepancias extremas que algunos alumnos presentaron 
entre los resultados del examen y los de la evaluación continua, y aun entre preguntas de contenido similar dentro del propio examen, nos hacen sospechar que el uso de medios ilícitos puede haber sido aún mayor del que pudimos demostrar.

El elevado porcentaje probado de uso de medios ilícitos y el hecho que muchos de los alumnos "cazados" negaran la evidencia o, aun admitiéndola, se creyeran igualmente merecedores del aprobado son, como mínimo, tan preocupantes como que muchos de los presentados hubiesen decidido comparecer a ésta y otras pruebas del curso con la expectativa de superarlas sin haberse preocupado siquiera por conseguir los materiales necesarios para prepararlas. Más aún, si la incapacidad para gestionar resultados negativos entre el alumnado centennial es ya notable en enseñanza presencial, el poder que las redes sociales dan al usuario para crear experiencias de uso sin emoción negativa alguna fortalece aún más la idea de que no se puede suspender una asignatura online y, como quedó patente en la revisión del examen y la Encuesta General de la Enseñanza, la incapacidad para aceptarlo y asumir cualquier tipo de responsabilidad personal cuando sucede.

Como anticipó la UOC hace ya dos décadas, en fin, una educación online de calidad pasa inevitablemente por contar con la normativa y los medios técnicos y humanos adecuados para desarrollarla; pero también-al menos, en una mayoría de asignaturas - por la existencia de mecanismos de verificación presencial de los conocimientos adquiridos. Cuando alguno de estos pilares falla o está ausente, como ha ocurrido estos meses en UniOvi, quizá podamos salir del paso, acallar las críticas de los estudiantes a base de aprobados, e incluso creernos que hemos sido capaces de proporcionar, de la noche a la mañana y a coste cero, una educación online verdadera y de calidad. Sin embargo, cuanto más tardemos en salir de la autocomplacencia y darnos cuenta de que la enseñanza online es otra cosa y cuesta mucho dinero, menos posibilidades tendremos de proporcionarla la próxima vez que haya que suspender la actividad presencial.

\section{Referencias}

Menéndez-Otero, C., R. Serrano-González, y I. Gil-Naveira. (2018). "Ensuring uniformity of content and assessment in multi-section, on-site ESP university courses through ICT". Revista de Lenguas para Fines Específicos, 24.2, pp. 9-26, ojsspdc.ulpgc.es/ojs/index.php/LFE/article/view/1008. [Acceso: $10 / 07 / 20]$

Ministerio de Universidades de España. (2020). "Borrador para Consulta: Estatuto del Personal Docente e Investigador”. En Federación de Educación CC.OO.

Madrid, www.feccoo-

madrid.org/e89380a282a20e8f64eb380c1711de6c000063.pdf. [Acceso: 05/07/20]

Ortega y Gasset, J. (1931). "Un aldabonazo". Crisol, 9 de septiembre. En SegundaRepublica.com, www.segundarepublica.com/index.php?opcion=7\&id=39. [Acceso: 25/06/20]

Real Decreto 463/2020, de 14 de marzo, por el que se declara el estado de alarma para la gestión de la situación de crisis sanitaria ocasionada por el COVID-19. Boletín Oficial del Estado, 67, pp. 25390-25400, www.boe.es/boe/dias/2020/03/14/pdfs/BOE-A-2020-3692.pdf. [Acceso: 24/06/20]

Scott, T. (dir.) (1986). Top Gun. Ídolos del aire. Paramount Pictures.

Vallespín, I. (2020). "Lo que hacen las universidades no se puede llamar educación 'online"”. El País, 12 de junio, elpais.com/educacion/2020-06-11/lo-quehacen-las-universidades-no-se-puede-llamar-educacion-online.html. [Acceso: 10/07/20]

*Autor de contacto: Carlos Menéndez Otero, menendezocarlos@uniovi.es

\section{MAGISTER}

Vol. 32. Núm. I: (2020). Sección extraordinaria 